\title{
Un nouvel objet pour la didactique du français : l'analyse des échanges en ligne
}

\author{
François Mangenot, \\ Lidilem, Grenoble 3
}

\section{Introduction}

Les situations pédagogiques lors desquelles tout ou partie de la communication s'effectue à travers le réseau Internet sont de plus en plus répandues, qu'il s'agisse de formation d'enseignants à distance (par exemple, les master FLE en ligne de Grenoble ou du Mans), d'échanges exolingues en ligne (Degache \& Mangenot, 2007) ou encore de travail collectif en complément d'une formation en présentiel (dispositifs dits «hybrides », cf. Charlier, Deschryver, Peraya, 2006). La didactique du français, qu'il s'agisse du FLM ou du FLE, n'est évidemment pas la seule à s'intéresser à ces nouvelles formes de communication pédagogique : les sciences de l'éducation, les sciences de l'information et de la communication (SIC), l'informatique (avec le sous domaine des Environnements interactifs pour l'apprentissage humain), voire la psychologie des apprentissages étudient également, sous des angles et avec des paradigmes de recherche divers, ces situations d'apprentissage instrumenté. Les sciences du langage et la didactique (du FLE, du FLM, des langues) possèdent cependant plusieurs atouts : elles analysent depuis longtemps les interactions en classe ; elles s'intéressent également aux interactions exolingues (Matthey, 2003); elles participent enfin, en concurrence - ou en interdisciplinarité - avec les SIC, au champ d'étude de la communication médiatisée par ordinateur (CMO), dont Jacques Anis (1998) est le pionnier en France ${ }^{1}$. Ces précédents lui donnent, on le verra, un regard spécifique sur ce domaine. Mais on examinera tout d'abord, de manière plus générale, l'intérêt de prendre la communication pédagogique médiatisée par ordinateur $\left(\mathrm{CPMO}^{2}\right)$ pour objet d'étude, puis quelques questions de recherche émergentes.

\section{Spécificités et apports des échanges en ligne}

L'intérêt pour la CPMO se fonde sur un présupposé fort, celui qui pose que le véritable apport d'Internet à la formation tient plus à sa dimension horizontale (échanges, mutualisation, réseaux sociaux, communautés de pratiques, etc.) qu'à sa dimension verticale transmissive (de loin la plus répandue, comme le constatent Albero et Thibault, 2004). On abordera ici quatre dimensions de la CPMO qui ne s'excluent pas les unes les autres : la contribution qu'elle peut apporter à la conception de dispositifs innovants, le lien social qu'elle permet d'assurer dans le cas des formations tout à distance, la mise en relation de locuteurs de langues différentes et la réflexivité que permet l'écrit asynchrone partagé.

\subsection{Des dispositifs innovants}

La Formation ouverte et à distance (FOAD) peut être considérée comme un secteur en voie de structuration scientifique (surtout en sciences de l'éducation), parallèlement à son implication pratique dans le champ de la formation professionnelle. Les notions de « dispositif», d' " approche dispositive », d'accompagnement pédagogique, d'autonomie et d'autoformation sont au cœur des investigations menées par les chercheurs de ce champ.

En langues, de plus en plus de dispositifs hybrides sont proposés aux étudiants non spécialistes (Degache \& Nissen, 2007) et peuvent donc faire l'objet d'études empiriques. Charlier, Deschryver \& Peraya (2006) définissent ainsi l'hybridation : « des dispositifs articulant à des degrés divers des phases de formation en présentiel et des phases de formation à distance, soutenues par un environnement technologique comme une plate-forme de formation »; ils soulignent par ailleurs le caractère innovant de tels dispositifs. L'innovation peut se jouer à différents niveaux : 
- Un assouplissement des contraintes spatio-temporelles et ainsi la possibilité de toucher de nouveaux publics n'ayant pas le temps de se déplacer une ou deux fois par semaine. Cet argument vaut notamment pour l'enseignement universitaire des langues aux spécialistes d'autres disciplines.

- Une individualisation plus grande de la formation: les apprenants désireux de s'investir davantage peuvent par exemple se voir offrir des tâches et ressources supplémentaires en ligne.

- Une diversification des formes d'expression : en classe, on communique le plus souvent à l'oral, par petits groupes ou en grand groupe. A distance, il est possible d'avoir une communication écrite collective débouchant sur une socialisation des productions écrites.

- Une place plus importante donnée à l'autoformation, à condition bien sûr que le public soit suffisamment autonome pour travailler en dehors de la présence de l'enseignant.

\subsection{Le lien social à distance}

Dans le cas de formations entièrement à distance, comme c'est le cas de plusieurs masters de FLE (Grenoble, Rouen, Le Mans), Internet permet d'une part de créer un lien plus interactif entre les enseignants et les étudiants, d'autre part et surtout d'établir un lien social entre les étudiants. On s'est assez rapidement rendu compte, à la fin des années 1990, que la faible participation des étudiants aux premiers forums qui accompagnaient les formations provenait plus d'un tutorat insuffisant et d'un manque d'objectifs fixés à ces forums que d'une réticence à échanger via Internet. Dès que des tâches collectives tirant parti de l'outil ont été proposées, la participation s'est fortement accrue et, à côté d'échanges de nature socio-cognitive, on a commencé à observer des phénomènes d'ordre socio-affectif et l'établissement d'un lien social (Develotte \& Mangenot, 2004). Engagé depuis 1999 dans la migration vers Internet d'un master de FLE auparavant délivré par correspondance sous forme traditionnelle, Mangenot $(2002,2003)$ a pu analyser les échanges écrits asynchrones qui se faisaient jour dans ce cadre et notamment mettre en relation les tâches proposées avec la nature des échanges entre pairs et avec le tuteur.

\subsection{Communication exolingue pour l'appropriation des langues}

S'il est un domaine où les échanges en ligne ont tendance à se multiplier, c'est bien celui de l'apprentissage des langues. La tradition du «pen friend» existait depuis longtemps, mais Internet a permis de revivifier ce genre de pratiques. S'il est toujours possible de faire communiquer des apprenants en mode un à un, avec les Tandems par Internet, de nombreux projets amenant des groupes à échanger en ligne de manière institutionnelle se sont développés ces dernières années: centrés parfois sur l'intercompréhension à l'intérieur d'une famille de langues (Galanet), souvent sur la pratique à parité de deux langues, visant presque toujours un échange au plan culturel, impliquant dans certains cas de futurs enseignants de langue, ces projets sont d'une grande diversité et on se contentera ici de renvoyer à deux recueils consacrés à cette question : Online Intercultural Exchange (O'Dowd, 2007) et Lidil 36, Échanges exolingues via Internet et appropriation des langues-cultures (Degache \& Mangenot, 2007). Plusieurs thèses, qui ont obtenu la qualification en sciences du langage, ont déjà été soutenues sur ce sujet.

\subsection{L'écrit numérique source de réflexivité}

Dans le prolongement de l'approche anthropologique de Goody, Lévy (1990) a qualifié les TIC de «technologies intellectuelles». Plus concrètement, des chercheurs anglais en sciences cognitives, Sharples \& Pemberton (1990), ont relevé les divers modes d'extériorisation de la cognition liés aux différents artefacts, du post-it aux assistants logiciels à l'écriture. Dans cette même optique, Lamy \& Goodfellow (1998) parlent de «conversations réflexives [...] par conférence asynchrone » et Mangenot (2004) souligne deux caractéristiques particulièrement intéressantes [des forums]: la souplesse chronologique qu'autorise le temps différé et la permanence de l'écrit qui fait du forum l'équivalent d'un 
texte en perpétuelle voie d'enrichissement. Cette permanence, liée à l'accessibilité, permet de parler à la fois d'extériorisation et de partage de la cognition.

C'est dans cet esprit qu'il est de plus en plus fréquent d'accompagner les stages professionnels ou l'entrée en fonction des futurs enseignants (ou enseignants débutants) par des forums ou des blogs ayant pour objectifs :

- de lier le terrain avec la réflexion didactique et de faire ainsi adopter une attitude épistémique par rapport aux problèmes rencontrés sur le terrain (Martin, 2003).

- de favoriser la construction d'une identité professionnelle (Soubrié, 2007).

- d'encourager la mutualisation et l'entraide (ou la « collégialité », Nault \& Nault, 2003).

Outre la formation d'enseignants, le réseau permet de mener des projets d'écriture avec des élèves de l'école primaire ou du collège ; dans ce cas, l'outil sert avant tout à valoriser et socialiser les écrits, dans le même esprit que ce qui se pratique dans les Ateliers d'écriture. Il ne semble pas encore y avoir beaucoup de recherches s'intéressant à ce dernier cas de figure.

Ayant ainsi un peu mieux caractérisé notre objet, les échanges en ligne, nous allons maintenant passer en revue quelques questions de recherche.

\section{Questions de recherche}

La formation à distance constituait déjà un objet de recherche avant l'émergence du réseau Internet. Mais l'instrumentation de la communication a rendu plus nécessaires les approches pluridisciplinaires. L'on peut ainsi distinguer des questions de recherche préexistantes au réseau Internet de celles qui abordent plus spécifiquement les situations pédagogiques collectives instrumentées. Précisons que cette partie ne prétend pas à l'exhaustivité.

\subsection{Questions anciennes revisitées par la CPMO}

On ne fera qu'évoquer d'une part la théorie de la distance transactionnelle, de M.G. Moore (bien décrite et opérationnalisée par Jézégou, 2007), et celle de l'industrialisation de la formation (Moeglin, 1998), ancrée pour l'une en sciences de l'éducation, pour l'autre en SIC. Ces deux théories ont le mérite de resituer la formation à distance dans un contexte institutionnel et sociopolitique plus large que les questions de CPMO, mais elles n'offrent que peu d'accroche pour le chercheur en didactique des langues.

A l'inverse, deux courants importants de la didactique des langues analysent les échanges en ligne. Ainsi, les acquisitionnistes anglo-saxons (Second Language Acquisiton Research) ont-ils conduit de nombreuses études autour de questions liées aux spécificités des situations de CPMO par rapport aux situations présentielles. Trois questions ressortent fréquemment, que nous ne ferons qu'évoquer en renvoyant à une référence, celle de la prise de parole plus aisée et mieux répartie (Kern, 1995 ; Fitze, 2006), celle des variations entre langue orale, langue écrite et langue de la CMO (Kern, 1995), celle de la richesse lexicale (Fitze, 2006), celle de la négociation du sens (Vandergriff, 2006).

De même, la question de l'acquisition d'une compétence interculturelle a-t-elle fait l'objet de plusieurs recherches récentes portant sur les échanges en ligne. Aux Etats-Unis, un courant est même apparu, avec la publication d'un ouvrage éponyme, l'Internet-mediated Intercultural Foreign Language Education (Belz \& Thorne, 2006). Les auteurs de cet ouvrage se penchent souvent sur des épisodes de communication manquée ( « missed communication ») et tentent de modéliser les conditions d'un dialogue interculturel efficace. Dans le même esprit, Mangenot \& Tanaka (2007), s'appuyant sur la notion d'ethos communicatif, montrent comment certains malentendus potentiels entre étudiants japonais et français peuvent être désamorcés - voire explicités - par les coordonnateurs de l'échange. 


\subsection{Questions nouvelles}

Les trois principales questions de recherche nouvelles suscitées par les échanges collectifs en ligne sont l'effet des outils de communication, le tutorat en ligne et les formes de travail collectif.

L'artefact technologique, on l'a déjà évoqué en citant Goody, n'est évidemment pas neutre par rapport aux pratiques langagières. Il est alors pertinent d'essayer de modéliser l'interrelation (également appelée " affordance ») entre les caractéristiques technologiques, les interactions verbales et les tâches réalisées par les apprenants, comme le font Lamy \& Hampel (2007, p. 33) dans un tableau où ces trois paramètres sont représentés sous forme de cercles entrecroisés. Cette approche est forcément pluridisciplinaire : les sciences du langage apportent leur connaissance des interactions verbales, l'informatique et l'ergonomie leurs analyses de la technologie, les SIC l'analyse des usages, les sciences de l'éducation ou la didactique des langues les typologies de tâches et d'objectifs. A noter l'intéressante notion de «culture-of-use », développée par Thorne (2006, p. 21), qui souligne que les outils de communication, en tant qu'artefacts culturels, prennent différentes significations et font l'objet d'usages différents (notamment en termes de genre) selon les communautés qui les utilisent; cet auteur cite des exemples de communication ratée du fait de l'utilisation d'un outil (le courriel) ressenti par les étudiants américains comme inadapté aux échanges entre pairs.

Le tutorat en ligne, pour sa part, s'il partage quelques caractéristiques avec d'autres formes d'accompagnement en face à face, présente néanmoins de fortes spécificités, notamment sur le plan temporel et sur celui du mode principal d'expression, la langue écrite. De nombreux travaux analysent ainsi le discours tutoral, que ce soit pour y repérer les différentes fonctions qu'il remplit (Denis, 2003), pour analyser la manière dont chacun s'efforce de créer un lien social (Develotte \& Mangenot, 2004), ou encore, de manière plus praxéologique, pour déterminer quel impact sur l'apprentissage ont différentes modalités d'intervention tutorale (Quintin, 2007).

L'étude des apprentissages collectifs en réseau, enfin, est l'objet de tout un champ de recherche très développé dans le monde anglo-saxon, le Computer-supported Collaborative Learning (CSCL). Contentons-nous ici de signaler que Dejean-Thircuir \& Mangenot (2006) critiquent l'omniprésence, au plan de la recherche, de la question de la collaboration (très exigeante pour les étudiants comme pour les tuteurs), au détriment de l'analyse d'autres formes d'apprentissage collectif comme la mutualisation ou la discussion.

\section{Quelques atouts des recherches appuyées sur les sciences du langage}

Il y a une douzaine d'années, des chercheurs en technologies éducatives relevant du champ du CSCL (cf. supra) avaient suggéré d'aborder les apprentissages collectifs médiatisés à partir d'une analyse qualitative des interactions verbales :

A promising possibility for collaborative learning research therefore is to exploit selective branches of linguistics research on models of conversation, discourse or dialogue to provide a more principled theoretical framework for analysis. [Dillenbourg et al., 1996, p. 203]

Leur hypothèse était que l'analyse des interactions pouvait permettre de caractériser les situations lors desquelles la collaboration se révélait efficace, plus sûrement qu'une approche expérimentale cherchant à contrôler le maximum de variables et à établir des rapports de cause à effet entre d'autres variables définies a priori. Cependant, la grande majorité des études qui ont emprunté cette voie (par exemple, Quintin, 2007) se sont appuyées sur l'analyse de contenu quantitative (ACQ), méthodologie impliquant «deux opérations fondamentales, la précatégorisation thématique des données textuelles, et leur traitement quantitatif, généralement informatisé » (Charaudeau \& Maingueneau (2002, p.39); on notera que ces auteurs opposent cette méthodologie à l'analyse du discours. De Wever et al. (2006) se livrent à une revue critique de quinze recherches consacrées à l'étude des forums pédagogiques et utilisant la méthodologie de l'ACQ; en réaction à cette étude, Mangenot (2007) remarque que la plupart des cadres 
théoriques passés en revue relèvent de la dimension cognitive ou socio-cognitive et émet l'hypothèse que l'ACQ se prête moins bien que des approches plus linguistiques à l'examen de phénomènes complexes liés à la dimension socio-affective ou au contexte socio-culturel, dans la mesure où elle se contente de coder et compter des énoncés au lieu de les analyser de manière plus fine.

L'on peut ainsi avancer que l'apport des sciences du langage se situerait plutôt du côté du qualitatif et de démarches ethnométhodologiques, l'idée étant d'observer, de décrire et de chercher à comprendre les logiques (les «méthodes ») des acteurs, éventuellement de parvenir à certaines modélisations, plutôt qu'à essayer de prouver l'efficacité de telle ou telle situation de collaboration à distance. Par manque d'espace, on se contentera ici de trois rapides illustrations, qui ne prétendent pas épuiser l'apport des sciences du langage à l'analyse des interactions en ligne.

L'approche «interactionniste cognitiviste » en didactique des langues, telle que proposée par Matthey (2003), peut assez aisément être reprise, plus ou moins fidèlement, pour l'analyse des interactions en ligne (Lamy \& Goodfellow, 1998), notamment dans le cas d'échanges plurilingues (Degache \& Tea, 2003). Rappelons que Matthey développe « une théorie de l'acquisition des langues secondes basée sur l'analyse d'interactions concrètes et dans lesquelles on cherche à observer des transmissions et des constructions de savoirs. » (p.136).

Anis (1998) a sans doute été le premier linguiste français à appliquer à des échanges électroniques, en l'occurrence des conversations par Minitel, un certain nombre de notions apportées par l'analyse conversationnelle; il complétait cet apport par une approche sémiolinguistique, portant une grande attention aux nouveaux « usages grapho-linguistiques ». Dans le domaine éducatif, il est tout à fait possible de s'appuyer sur une telle approche, pour observer par exemple la manière dont se structurent les interactions (Celik, 2007), dont se positionnent et se mettent en scène les acteurs les uns par rapport aux autres (Blandin, 2004), dont se jouent les questions de faces (Mangenot, 2004), notamment en situation interculturelle.

Concernant l'analyse du discours ${ }^{3}$, celle-ci peut être appelée à la rescousse pour caractériser les marques énonciatives permettant de repérer l'établissement d'une communauté d'apprentissage ou plus largement les marques caractérisant le lien socio-affectif (Develotte \& Mangenot, 2004); ou encore pour relever une source possible de malentendu dans les échanges interculturels liée à la question des genres de discours en ligne (Ware \& Kramsch, 2005, Thorne, 2006). Elle est également bien outillée pour repérer les ethos communicatifs (Kerbrat-Orecchioni, 2005) selon l'appartenance culturelle, voire la «dimension idioculturelle des micro-communautés d'apprentissage en ligne » (Dolci \& Spinelli (2007).

\section{Références}

Albero, B., Thibaut, F. (2004) Enseignement à distance et autoformation à l'université: au-delà des clivages institutionnels et pédagogiques? In Saleh, I., Bouyahi, S. (éds.) Enseignement ouvert et à distance, épistémologie et usages, p. 35-52. Paris : Lavoisier.

Anis, J. (1998) Texte et ordinateur, l'écriture réinventée ? Paris, Bruxelles : De Boeck université.

Belz, J.A. \& Thorne, S.L. (2006, ed.) Internet-mediated Intercultural Foreign Language Education. Boston: Thomson Heinle.

Blandin, B. (2004) La relation pédagogique à distance : que nous apprend Goffman? Distances et savoirs $\mathrm{n}^{\circ} 2-3$, p.357-381.

Celik, C. (2007) Les modalités du tutorat dans un campus électronique de maîtrise de français langue étrangère entièrement à distance. In Lamy, Mangenot, Nissen.

Charaudeau, P., Maingueneau, D. (2002, coord.) Dictionnaire d'analyse du discours. Paris : Seuil.

Degache, C. \& Mangenot, F. (2007, coord.) Lidil n³6, Echanges exolingues via Internet et appropriation des langues-cultures. Grenoble, Ellug. 
Degache, C., Nissen, E. (2007) Formations hybrides et interactions en ligne du point de vue de l'enseignant : pratiques, représentations, évolutions. In Lamy, Mangenot, Nissen.

Degache, C., Tea, E. (2003) Intercompréhension : quelles interactions pour quelles acquisitions ? Les potentialités du Forum Galanet. Lidil n²8, p.75-94.

Dejean-Thircuir, C. \& Mangenot, F. (2006) Tâches et scénario de communication dans les classes virtuelles. In Les Cahiers de l'Asdifle n $^{\circ}$ 17, p.310-321. Paris, Association de didactique du FLE.

Denis, B. (2003) Quels rôles et quelle formation pour les tuteurs intervenant dans des dispositifs de formation à distance? Distances et savoirs, Vol. 1, $\mathrm{n}^{\circ} 1$, p.19-46. Paris, CNED/Lavoisier.

Develotte, C. \& Mangenot, F. (2004) Tutorat et communauté dans un Campus numérique non collaboratif. Distances et savoirs Vol. 2 n²-3, p.309-333. Paris, CNED/Lavoisier.

De Wever, B., Schellens, T., Valcke, M., Van Keer, H. (2006) Content analysis schemes to analyze transcripts of online asynchronous discussion groups: A review. Computers \& Education 46 (2006), p.6-28.

Dillenbourg, P., Baker, M., Blaye, A., O'Malley, C. (1996) The Evolution of Research on Collaborative Learning. In Spada, E. \& Reiman, P. (éds.) Learning in Humans and Machines: Towards an interdisciplinary learning science. p.189-211. Oxford: Elsevier.

Dolci, R., Spinelli, B. (2007) La dimension idioculturelle des micro-communautés d'apprentissage en ligne. Lidil n³6, p. 69-92.

Fitze, M. (2006) Discourse and participation in ESL face-to-face and electronic written conferences. Language Learning and Technology, Vol. 10 Num.1, 66-86. http://lit.msu.edu

Herring, S. (2004) Computer-Mediated Discourse Analysis, An approach to Researching Online Behavior. In Barab, S., Kling, R. \& Gray J. (eds.) Designing for Virtual Communities in the Service of Learning, 338-376. Cambridge University Press.

Jézégou, A. (2007) La distance en formation, Premier jalon pour une opérationnalisation de la théorie de la distance interactionnelle. Distances et savoirs vol. 5, n³/2007, p. 341-366.

Kerbrat-Orecchioni, C. (2005) Le discours en interaction. Paris : Armand Colin.

Kern R. G. (1995) Restructuring classroom interaction with networked computers: effects on quantity and characteristics of language production. The Modern Language Journal 79, p.457-476.

Lamy, M.-N. \& Goodfellow, R. (1998) Conversations réflexives dans la classe de langues virtuelle par conférence asynchrone. $A L S I C$ Vol. 1, n², p.81-99. http://alsic.org

Lamy M.-N., Hampel R. (2007) Online Communication in Language Learning and Teaching. Basingstoke (U.K.), Palgrave Macmillan.

Lamy, M.-N., Mangenot, F., Nissen, E. (2007) Actes du colloque EPAL (Echanger pour apprendre en ligne), Grenoble 7-9 juin 2007. http://w3.u-grenoble3.fr/epal/actes.htm

Lévy, P. (1990) Les technologies de l'intelligence, L'avenir de la pensée à l'ère informatique. Paris : Éditions La Découverte.

Mangenot, F. (2002) Forums et formation à distance : une étude de cas. Education permanente 152, p.109-119.

Mangenot, F. (2003) Tâches et coopération dans deux dispositifs universitaires de formation à distance. ALSIC Vol. 6, num. 1, p.109-125. http://alsic.org

Mangenot, F. (2004) Analyse sémio-pragmatique des forums pédagogiques sur Internet. In Salaün, J.-M. \& Vandendorpe, C. (coord.), Les défis de la publication sur le Web : hyperlectures, cybertextes et méta-éditions, $\mathrm{p}$. 103-123. Villeurbanne, Presses de l'Enssib.

Mangenot, F. (2007) Analyser les interactions pédagogiques en ligne, pourquoi, comment? In Gerbault, J. (coord.) La langue du cyberespace : de la diversité aux normes, p. 105-120. Paris : L'Harmattan.Mangenot F., Nissen E. (2006) Collective activity and tutor involvement in e-learning environments for language teachers and learners. Calico Journal Vol. 23 (3), p.601-622.

Mangenot, F., Tanaka, T. (2007) Les enseignants de langue comme médiateurs entre deux cultures dans les interactions en ligne : le cas d'un échange franco-japonais. In Lamy, Mangenot, Nissen. 
Martin, D. (2003) Forum de discussion en formation des maîtres : apprentissage de la délibération collégiale. In Deaudelin \& Nault (coord.) Collaborer pour apprendre et faire apprendre. La place des outils technologiques, p. 103-119. Presses de l’Université du Québec.

Matthey, M. (2003, 2ème éd.) Apprentissage d'une langue et interaction verbale. Bern : Peter Lang.

Moeglin, P. (dir., 1998) L'industrialisation de la formation. Etat de la question. Paris : CNDP.

Mourlhon-Dallies, F. (2007) Communication électronique et genres du discours. Revue en ligne Glottopol Vol. 10 (2007). http://www.univ-rouen.fr/dyalang/glottopol

Nault G., Nault T. (2003) Communauté virtuelle : un soutien pour des enseignants novices en cheminement vers la collégialité. In Deaudelin \& Nault (coord.) Collaborer pour apprendre et faire apprendre. La place des outils technologiques, p. 191-210. Presses de l’Université du Québec.

O’Dowd, R. (2007, ed.) Online intercultral exchange, An Introduction for Foreign Language Teachers. Clevedon, Buffalo, Toronto: Multilingual Matters.

Quintin, J.-J. (2007) Accompagnement d'une formation asynchrone en groupe restreint : modalités d'intervention et modèles idiosyncrasiques des tuteurs. In Lamy, Mangenot, Nissen.

Sharples M., Pemberton L. (1990) Starting from the Writer: Guidelines for the Design of User-centred Document Processors. Computer Assisted Language Learning Vol. 2 / 1990, p.37-57. Oxford, Intellect.

Soubrié, T. (2007) Images de soi dans un blog professionnel d'enseignants stagiaires. In Lamy, Mangenot, Nissen.

Thorne, S.L. (2006) Pedagogical and Praxiological Lessons from Internet-mediated Intercultural Foreign Language Education Research. In Belz, J.A. \& Thorne, S.L. (dir.) Internet-mediated Intercultural Foreign Language Education. Boston: Thomson Heinle. p.2-30.

Vandergriff, I. (2006) Negotiating common ground in computer-mediated versus face-to-face discussion. Language Learning and Technology, Vol. 10 Num.1, p.110-138. http://ltt.msu.edu

Ware, P. D., Kramsch, C. (2005) Toward an intercultural stance: Teaching German and English through telecollaboration. The Modern Language Journal, 89(2), p. 190-205.

\footnotetext{
${ }^{1}$ Voir notamment le numéro 104 de Langage et société et le numéro 8 des Carnets du Cediscor.

${ }^{2}$ Ce sigle présente l'avantage d'être plus neutre et plus englobant quant aux objectifs et modalités de la communication que, par exemple, Computer Supported Collaborative Learning, un champ pluridisciplinaire très investi par les chercheurs anglo-saxons en sciences de l'éducation et informatique.

${ }^{3}$ On remarquera que l'analyse de discours «à la française » ne correspond pas à ce que les anglo-saxons appellent «Discourse analysis»; Herring (2004), notamment, propose une approche, la Computer-Mediated Discourse Analysis, qui n'exclut ni l'analyse de contenu quantitative, ni des méthodes plus linguistiques.
} 